\title{
Accelerometer Based Control of Robotic Arm
}

\author{
Y.V. Sawant, S.M. Karmuse, N.B. Kapase \\ Department of Electronics Engineering, D.K.T.E'S Textile and Engineering Institute, India
}

\begin{tabular}{l} 
Article Info \\
\hline Article history: \\
Received Apr 28, 2016 \\
Revised Aug 4, 2016 \\
Accepted Aug 18, 2016 \\
\hline
\end{tabular}

\section{Keyword:}

Accelerometer

Bluetooth

FRDM-KL25Z

Robotic arm

\begin{abstract}
Robots are very smart and interactive machines that can be programmed and used in many areas such as industry, manufacturing, production lines, household or health, etc. These robots can perform hard, dangerous, but accurate work to facilitate our life and to increase the production. Humans are trying to minimize the time required to do the similar kind of jobs. The task in which picking \& placing of an object is to be done, can be carried out with the help of robots. These robots can minimize the human efforts. The robotic arms can be used in the industries to assemble the small parts,or in chemical industries to pick and place small types of object such as tablets. Artificial robotic arms thus can be acquainted to control gestures. In this paper an idea of controlling robotic arm is implemented using an accelerometer which was placed on the human hand, capturing its behavior (gestures and postures) which were then transmitted at some distance and the robotic arm assembly connected wirelessly was moved accordingly.
\end{abstract}

Copyright $\left(C_{0} 2016\right.$ Institute of Advanced Engineering and Science. All rights reserved.

\section{Corresponding Author:}

Y.V Sawant,

D.K.T.E'S Textile \& Engineering Institute,

Department of Electronics Engineering,

Ichalkaranji, India.

Email: yogitas165@gmail.com

\section{INTRODUCTION}

Robotics is field where we are having lots of scope for innovations and to do something innovative. It is very interactive field with lot of technological variations. With each passing day the gap between machines and humans is getting reduced with the introduction of new technologies to facilitate the standard of living. In robotics research, robotic arms are used with unique features and design criteria. Most of the robotic arms are controlled by using accelerometer sensor and with an artificial intelligent algorithm. The robotic arms are able to achieve reliable distant communication.User friendly robot control is an important open area of research to robotics. Robotic systems are now a day the vital part of almost all the industries. In industries, a robotic arm perform various tasks such as welding, trimming, picking and placing small objects etc [1]. The biggest advantage of these arms is that they can work in hazardous areas and also in the areas which cannot be accessed by humans.

Gestures played a crucial role in many commutation applications where human interaction is essential. Gestures can be captured with the help of an accelerometer, however, with the development of smartphone its independent usage has been rendered useless. Many researchers worked on the implementation of robotic arm. MEMS accelerometer is used to give the hand gestures. MEMS means micro electro mechanical system which has 3 axis of $\mathrm{x}, \mathrm{y}, \mathrm{z}$ and a power supply port with ground is fabricated [2].

Most of industrial robots are still programmed using the typical teaching process. An industrial robot system was designed using two low-cost and small 3-axis wireless accelerometers. These accelerometers were attached to the human arms, capturing its behavior (gestures and postures). Artificial Neural Network trained with a back-propagation algorithm was used to recognize arm gestures and postures, which would be used as input in the control of the robot. The aim was that the robot starts the movement almost at the same time as the user starts to perform a gesture [3]. An idea was of using robotic arm design 
software to design a $6-\mathrm{DOF}$ (degree of freedom) virtual robotic arm which in turn control an actual robotic arm was implemented [4]. The hand gesture recognition system for cutting weeds in the crop field was designed by using MEMS accelerometer and PIC micro controller [5]. The motion technology was analyzed by the author to capture gestures through an android Smartphone with an inbuilt accelerometer and a Bluetooth module to control the kinetics of a robot [6]. A robust MEMS based Gesture Controlled Robot can be designed for recognizing hand motion based gesture interfaces. An innovative Formula for gesture recognition was developed for identifying the distinct action signs made through hand movement [7] [8]. Flex sensors along with accelerometer attached to hand or can give better accuracy in gesture recognition[9] and gyro-meter with accelerometer can improve the stability and to detect the rotational gesture of human arm [10].

The robotic arms are available in the market, but the wireless implementation using accelerometer is rarely used. Robotic arms are with the buttons or joysticks are used for controlling in industries. Our aim was to develop a model which will be efficiently used to minimize human efforts. In this paper we explored a system where an embedded accelerometer was used to track the movements and a robotic arm assembly was controlled by micro controller.

\section{PROPOSED METHOD}

The hardware part of the system was divided into two sections as transmitter section and receiver section as follows,

\subsection{Transmitter section}

In the transmitter section, sensor interfacing was main thing.For this FRDMKL25Z kit was

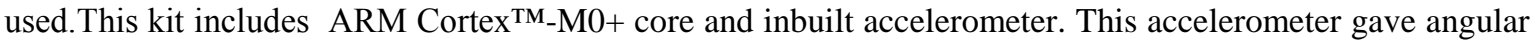
position values, these values are in $\mathrm{X}, \mathrm{Y}, \mathrm{Z}$ co-ordinates in the range of 0.00 to 1.00 . In wireless mode Bluetooth was used for transmitting those values. Further these values are received at receiver section.

\subsection{Receiver Section}

In the receiver section 8051 micro controller was used for controlling the motors. The Bluetooth at the receiver side will received the $\mathrm{X}, \mathrm{Y}, \mathrm{Z}$ values and those values are transmitted to controller.According to these values the motors will rotate and the movement of robotic arm will take place. To drive the motors driver circuitry was used. The block representation of the system is shown in figure 1 and Figure 2.

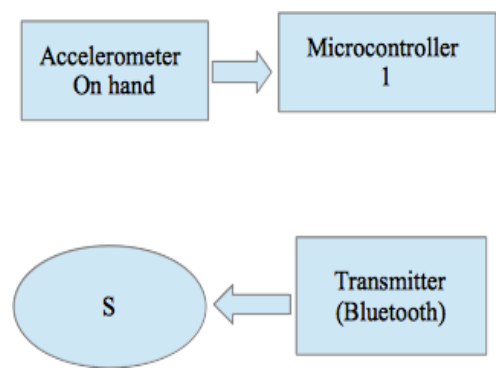

Figure 1. Transmitter section

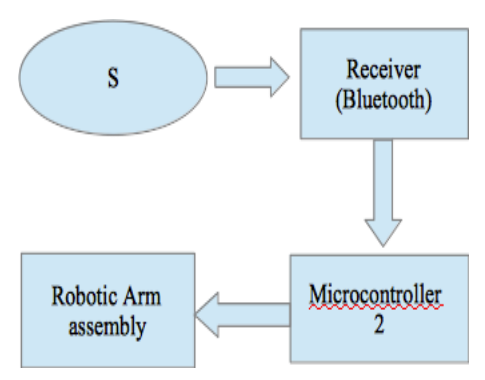

Figure 2. Receiver Section

\section{HARDWARE DESIGN TECHNIQUES}

Accelerometer sensors are very useful in digital technology. Smart phone, smart vehicles, automation industries where smart management is required are some areas where accelerometers are used.

\subsection{FRDM- K125Z kit}

Heart of this project is Accelerometer sensor. The MMA8451Q accelerometer sensor is embedded in FRDM-K125Z kit. This is a Freescale Freedom development platform where a set of software and hardware tools are used for evaluation and development. It is ideal for rapid prototyping of micro controllerbased applications. The Freescale Freedom KL25Z hardware, FRDM-KL25Z, is a simple, yet sophisticated

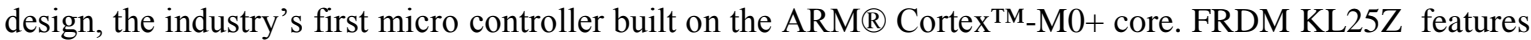
a KL25Z128VLK, a KL2 family device boasting a max operating frequency of $48 \mathrm{MHz}, 128 \mathrm{~KB}$ of flash, a 
full-speed USB controller, and loads of analog and digital peripherals. The on-board interfaces include an RGB LED, a 3-axis digital accelerometer, and a capacitive touch slider.

The FRDM-KL25Z ishown in Figure 3 is the first hardware that offers several options for serial communication. This kit is programmed using Mbed.org platform using the mbed online IDE, a free online code editor and compiler in which the code is written and compiled within a web browser.

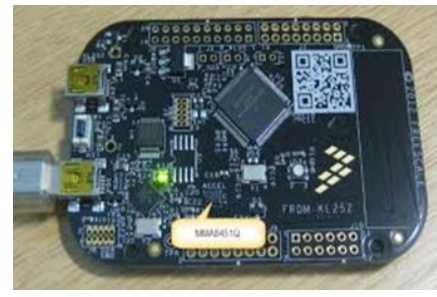

Figure 3. FRDM kit

In this system, the FRDM was mounted on the human arm and the movements of which are captured and converted into $\mathrm{x}, \mathrm{y}, \mathrm{z}$ co-ordinates.

\subsection{Bluetooth}

Bluetooth is a low cost wireless technology standard for exchanging data over short distances.In this work and $\mathrm{H} 05$ and $\mathrm{H} 06$ bluetooth modules were used.The micro controller communicates with AT serial commands with the module. The HC-06 has the 'full' firmware on it: many AT commands, and can be used as master or slave module.

\subsection{L293D Driver IC}

The L293D has been designed to provide bidirectional drive current of up to $1 \mathrm{~A}$ at voltage from $4.5 \mathrm{~V}$ to $36 \mathrm{~V}$. It Designed for to drive inductive loads such as relay, solenoid, DC and bipolar stepping motor, as well as high current/high voltage loads in positive supply application. Drivers are enabled in pairs with driver 1 and 2 enabled by 1,2 EN and driver 3 and 4 enable by 3,4 -EN. A VCC1 terminal, separate from VCC2, is provided for the logic inputs to minimize device power dissipation. The L293 and L293D are characterized for operation from $0^{\circ} \mathrm{C}$ to $70^{\circ} \mathrm{C}$.

In the proposed system it was used to drive the motors in robotic arm in angular directions because the controller outputs currents are insufficient to drive them.

\subsection{Micro controller}

It is 8-bit micro controller, means MC 8051 can Read, Write and Process 8 bit data. This is mostly used cost efficient micro controller in the robotics. The micro controller has built in ROM, RAM, Input Output ports, Serial Port, timers, interrupts and clock circuit. The I/O, memory, and on-chip peripherals of a micro controller are selected depending on the specifics of the target application. Since micro controllers are powerful digital processors, the degree of control and programmability they provide significantly enhances the effectiveness of the application. The micro controller has on chip peripheral devices. It was the controlling block in our system, to which all other components (Accelerometers, Motors, bluetooth modules etc.) are interfaced. Two such micro controllers were used in this project, one at the transmitting end and one at the receiving end. The micro controller was programmed through $\mu$ Vision's keil software.

\subsection{The robotic Arm assembly}

The robotic arm assembly is shown in figure below. This was the important part of the system which does angular movements for Pick and Drop task in the project. The robotic arm is equipped with a Gripper (for picking and placing the objects) and an Arm (for raising and lowering the objects), shown in Figure 5. Both the Arm and Gripper were equipped with DC Motor to control the movement. These movements were synchronized with the hand gestures of the user at transmitter side. The accelerometer was mounted on hand, which captures the hand gestures. The assembly was controlled at the receiver section by the second controller. The DC motors in the assembly were driven by the values sent through micro controller. L293D Driver assist here. 


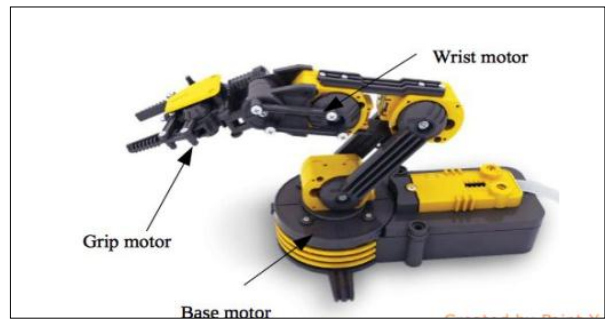

Figure 4. Robotic Arm Assembly

\section{SYSTEM IMPLEMENTATION}

When the power supply of both transmitter and receiver was turned $\mathrm{ON}$, then the Bluetooth device of transmitter will connect to the Bluetooth device of receiver section. The FRDM kit was mounted on the hand. As it moved in any direction,the X,Y,Z values of current position were changed. When the accelerometer was tilted in right direction it gives $X$ value (angular value) in positive sign and when the accelerometer is tilted in left direction then it gave value in negative sign and at that time $\mathrm{Y}$ value was approximately zero and $\mathrm{Z}$ value was approximately one in positive sign. When accelerometer was rotated by 180 degree then value of $\mathrm{z}$ become negative.

These values were received by another bluetooth in the receiver section. The second Micro controller selects the appropriate motor, out of four motors, wrist motor, grip motor or base motor present in the arm assembly depending upon the range of the values of $\mathrm{X}, \mathrm{Y}, \mathrm{Z}$ coordinates that has been received and that motor was driven. The flow chart of this rotation statistics is shown in figure below.

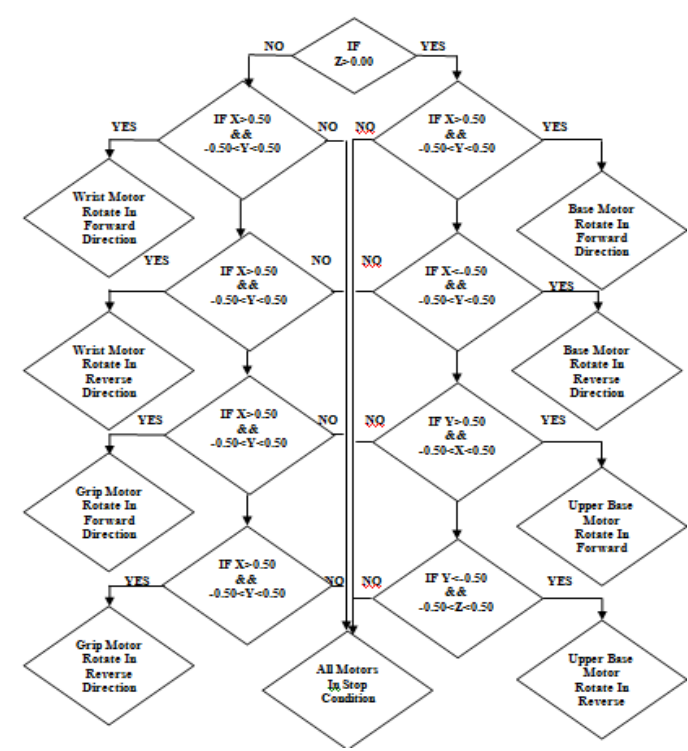

Flowchart 1. Motor rotation statistics

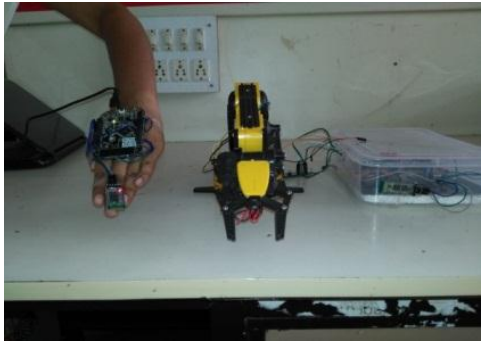

Figure 5a. Implementation of system

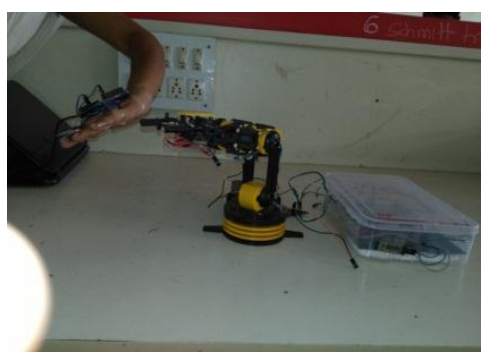

Figure 5b. Implementation of system 
The total system implementation is shown in figure $5 \mathrm{a}$ and $5 \mathrm{~b}$ where the movement of the arm assembly is shown in two different directions.

\section{RESULT AND DISCUSSION}

In this system, the proper angular movements of Robotic arm assembly was obtained according to the movement of accelerometer in all directions. The project showed the ability to grab the object and to place that object at any other place. In this work Bluetooth have been used for wireless communication. Bluetooth connection can not get a capture range more than 10m (for HC06) and which is a drawback as par as distance is considered. X-bee or RF modules which provides long distance communication can be used in place of bluetooth. Also use of camera at receiver side can facilitate the performance of the system This camera will help us to operate the robotic arm accurately. The higher resolution cameras will help us to get greater accuracy. For future modification android apps can be developed to run the system.

\section{CONCLUSION}

Robots play the vital role in human life where number of tasks can be performed with ease. Robots are having wide range of applications in various fields. Robots are well suited for the areas where human can't enter. The accelerometer based robotic arms can be used in industries where heated metal plates,tablets or other objects are lifted. Such type of systems can be also used to ease handicapped people to drive their wheelchair. This would be a measure application for the society.

\section{REFERENCES}

[1] Prof. R. W. Jasutkar, Ms. Shubhangi J. Moon, "A Real Time Hand Gesture Recognition Technique by using embedded device". International Journal of Advanced Engineering Sciences and Technologies, vol. 2, issue no.1, pp. 043-046 May 2005.

[2] Huang W, Cai X, Xu B, Luo L, Li X, Cheng Z,. Packaging effects on the performances of MEMS for high-G accelerometer with double-cantilevers. IEEE Conference on Sensors and Actuators A. 2008.

[3] Pedro Neto, J.and Norberto Pires, “Accelerometer-Based Control of an Industrial Robotic Arm", IEEE conference on Robot and Human Interactive Communication, 2009.

[4] Wong Guan Hao, Yap Yee Leck and Lim Chot Hun. "6-DOF PC Based robotic arm (PC-roboarm) with efficient trajectory planningand speed control” 2011 4th International Conference on Mechatronics (ICOM), 17-19 May 2011, Kuala Lumpur, Malaysia.

[5] K. Ashok Kumar and K. Thamizharasi, "Gesture Controlled Robot using MEMS Accelerometer for Eradication of Weeds" Indian Journal of Science and Technology, Vol 8(5), 460-465, March 2015.

[6] Monika Jain, Aditi, Ashwani Lohiya, Mohammad Fahad Khan, Abhishek Maurya, "Wireless gesture control robot: an analysis" International Journal of Advanced Research in Computer and Communication Engineering Vol. 1, Issue 10, December 2012

[7] Vivek Bhojak, Girish Kumar Solanki, Sonu Daultani "Gesture Controlled Mobile Robotic Arm Using Accelerometer" international journal of innovative research in science, engineering and technology vol. 4, issue 6, june 2015

[8] N.V.Maruthi Sagar, D.V.R.Sai Manikanta Kumar, N.Geethanjali describes in "Mems Based Gesture Controlled Robot Using Wireless Communication" International Journal of Engineering Trends and Technology (IJETT) Volume 14 Number 4 - Aug 2014.

[9] Abidhusain Syed, Zamrrud Taj H. Agasbal, Thimmannagouday Melligeri , Bheemesh Gudur, "Flex Sensor Based Robotic Arm Controller Using Micro Controller", Journal of Software Engineering and Applications, 2012, Vol 5, 364-366.

[10] Ariful Islam Bhuyan, Tuton Chandra Mallick, "Gyro-accelerometer based control of a robotic Arm using AVR micro controller", IEEE conference on Strategic Technology (IFOST), 2014. 


\section{BIOGRAPHIES OF AUTHORS}

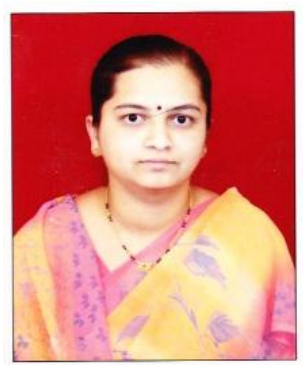

Mrs.Y.V.Sawant is working as assistant professor in Electronics department at D.K.T.E'S textile Engineering Institute, Ichalkaranji. She has completed her post graduation in Electronics

Engineering from Shivaji university,India. Her main areas of research interests are nonlinear control, Artificial neural networks, Image processing and Robotics.

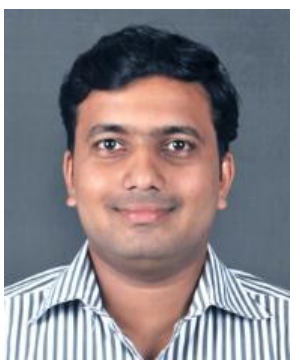

Mr Niraj B Kapase working as Assistant Professor in Electronics Department at DKTE's Textile and Engineering Institute Rajwada Ichalakranji. He has completed his post graduation form University of Surrey Guildford UK area of specialization is Mobile and Satellite Communications. His main area of interest and research is Embedded Systems design and Wireless Communications. 